\title{
Demystifying the success of empirical distributions in space plasmas
}

\author{
Kamel Ourabah $\mathbb{0}^{*}$ \\ Theoretical Physics Laboratory, Faculty of Physics, University of Bab-Ezzouar, USTHB, Boite Postale 32, El Alia, Algiers 16111, Algeria
}

(Received 23 February 2020; accepted 8 April 2020; published 4 May 2020)

\begin{abstract}
Motivated by the recent result by Davis et al. [Phys. Rev. E 100, 023205 (2019)] that velocity distributions of collisionless steady-state plasmas must follow superstatistics, we examine systematically the ability of superstatistics to account for observations of anomalous distributions in plasma physics. We consider the two possible scenarios: the case where empirical distributions are postulated to account for indirect measurements of dispersion relations and the case where direct in situ measurements of the distributions are available. In the former case, it is shown that the three universality classes of superstatistics allow one to account for measurements, in opposition to previous claims that such measurements constitute a signature of the specific class of Tsallis distributions. In the latter scenario, the two classes of $\chi^{2}$ superstatistics and lognormal superstatistics are shown to reproduce the profiles of typical observations. In particular, the class of lognormal superstatistics, ignored in the plasma physics literature, allows reproducing typical observations while overcoming severe limitations of the standard empirical distributions related to diverging moments. We further discuss how the superstatistical picture may open up new prospects for investigating thermodynamic properties of space plasmas, by virtue of the relationship between temperature fluctuations and the heat capacity, while still preserving the entropy extensivity.
\end{abstract}

DOI: 10.1103/PhysRevResearch.2.023121

\section{INTRODUCTION}

It is now widely acknowledged that statistical properties of space plasmas often depart from the canonical [read MaxwellBoltzmann (MB) or Jüttner] distributions. A number of empirical distributions have been introduced phenomenologically for that reason and, while their origin is debatable and remains obscure to some degree, their success in modeling space plasma phenomena is common knowledge. Among the most studied distributions, the kappa suprathermal and the Cairns nonthermal distributions play a privileged role. They constitute a substantial and increasing part of the plasma physics literature [1-7] and are, in some occasions, combined in various ways to form hybrid distributions [8-10]. The success of empirical distributions in modeling space plasma phenomena is not surprising since, after all, canonical distributions constitute a facet of equilibrium statistical mechanics, only applicable for systems in equilibrium. In the evolution of a system toward equilibrium, collisions play a fundamental role. At low-pressure conditions, e.g., in the solar wind, collisional events become negligible and the assumption that the system has reached thermal equilibrium becomes hardly justified. Hence, empirical distributions can be-and in general areregarded as a manifestation of a nonequilibrium steady state. Finding the steady state of a nonequilibrium system is a highly nontrivial matter because, in principle, it requires the knowledge of the entire past history of perturbations that the

\footnotetext{
*kam.ourabah@gmail.com

Published by the American Physical Society under the terms of the Creative Commons Attribution 4.0 International license. Further distribution of this work must maintain attribution to the author(s) and the published article's title, journal citation, and DOI.
}

system has undergone. One possible alternative to overcome this issue, particularly well suited for systems exhibiting local equilibrium, consists in describing the nonequilibrium system provided only one or a few extra parameters over those required to describe the system at equilibrium. One may think for instance of using only two parameters: a nonequilibrium temperature and a parameter measuring the distance from equilibrium. The formalism of superstatistics and the related concept of hyperensembles are centered around this very idea.

The main idea can nicely be illustrated by thinking of a simple system: that of a Brownian particle that moves in an inhomogeneous medium. The latter can safely be divided up into cells, each characterized by a sharp value of the temperature in such a way that, within each cell, the whole machinery of equilibrium statistical mechanics holds. As the particle moves from one cell to another, it "sees" temperature changes, ultimately produced by the complex dynamics of the environment. Provided that the particle stays long enough in each cell to thermalize, the long-term stationary probability distribution arises out of the canonical distribution, associated with each cell, averaged over the distribution of the temperature across the different cells. At the heart of this methodological attitude, there is the adiabatic Ansatz [11]: During its evolution, the system travels within its state space $X$ which is divided up into small cells, each characterized by a sharp value of some intensive quantity $\zeta$. Within each cell, the system is described by the conditional distribution $p(A \mid \zeta)$ to be found in a specific state $A \in X$. As $\zeta$ varies adiabatically from cell to cell, the joint distribution of finding the system with a sharp value of $\zeta$ in the state $A$ is $p(A, \zeta)=p(A \mid \zeta) p(\zeta)$, viz., the de FinettiKolmogorov relation. The resulting statistics $p(A)$ for finding the system in the state $A$ is obtained through marginalization and reads as a superposition of statistics, i.e., superstatistics:

$$
p(A)=\int p(A \mid \zeta) p(\zeta) d \zeta
$$


In plasma physics, one is usually interested in the velocity distribution, that is, $A \equiv v$, under a fluctuating (inverse) temperature, that is, $\zeta \equiv \beta$. While one locally has $p(v \mid \beta)$, corresponding to a Maxwellian distribution, the emergent distribution $p(v)$ may deviate from it in a way dictated by the specific class of fluctuations.

Superstatistics, as a formalism, was introduced in Ref. [12], but the logic behind it has a long tradition in statistical mechanics and similar Ansätze have previously been used in quite different contexts [13-17]. The formalism has enjoyed a considerable degree of success ranging from fields as disparate as high-energy physics [18,19] and cosmology [20,21] to mathematical finance [22] and power grid fluctuations [23], among many others [24-26]. However, in spite of the ubiquitous presence of noncanonical distributions in space and laboratory plasmas, this paradigm has had little attention so far in plasma physics. In this direction, we showed recently [27] that the nonthermal and suprathermal distributions can be mapped onto superstatistics. Later on, in a series of papers [28-30] Davis et al. offered a number of formal results paving the way for a more complete and rigorous implementation of this paradigm in plasma physics. In particular, in Ref. [29] they show that the single-particle velocity distributions of collisionless steady-state plasmas must follow superstatistics. Here, we take advantage of this result and (re)examine the ability of superstatistics to explain the behavior of space plasmas with particular attention given to the following aspects:

First, we point out that not all empirical distributions widely used in plasma physics have been directly observed but are often postulated to account for indirect measurements. This is the case for instance of the Cairns distribution [31] that was not directly observed but postulated ad hoc to account for the observation in the upper ionosphere of solitary electrostatic structures involving density depletions. One natural question then is whether the effect produced by such distributions on the observed mechanism is specific to them or whether other distributions, with a more transparent statistical origin, produce the same effect. Second, even when the distributions emerge from a direct observation, one relies on curve fitting to construct a "smooth" function that approximately fits the data. Here again, a natural question is whether other distributions, more justified from the statistical mechanics standpoint, can also do the job. Last but not least, owing to the difficulty in space plasmas of realizing in situ measurements of the temperature, the latter is usually measured indirectly through the observation of velocity distributions. We will demonstrate in the following that the superstatistical picture not only clarifies the ambiguity between the observed temperature and the mean temperature, but also, by virtue of the link between temperature fluctuations and the heat capacity, allows one to investigate the thermodynamic aspects of space plasmas, while preserving the entropy extensivity.

\section{NON-GAUSSIAN VELOCITY DISTRIBUTIONS FROM SUPERSTATISTICS}

To begin with, let us convince ourselves that velocity distributions similar to those widely used in modeling space plasmas emerge from temperature fluctuations. For this, consider a system made of smaller subsystems, each of them in thermodynamic equilibrium with an inverse temperature $\beta$. Each subsystem is considered large enough to obey statistical mechanics but has a different (inverse) temperature assigned to it, according to a probability density $f(\beta)$. In principle, there are infinitely many possible temperature distributions but it is known [38] that three fundamental classes of $f(\beta)$ arise as universal limit statistics in the majority of known superstatistical systems:

(a) $\chi^{2}$ superstatistics. In this case, the inverse temperature $\beta \equiv 1 / k_{B} T$ (henceforth, $k_{B}=1$ ) is distributed according to a $\chi^{2}$ distribution of degree $n$. That is,

$$
f(\beta)=\frac{1}{\Gamma\left(\frac{n}{2}\right)}\left(\frac{n}{2 \beta_{0}}\right)^{n / 2} \beta^{n / 2-1} e^{-\frac{n \beta}{2 \beta_{0}}},
$$

where $\beta_{0}$ is the average of $\beta$ and $n$ a positive parameter. The corresponding $d$-dimensional long-term velocity distribution (marginal distribution), i.e., Eq. (1), reads as

$$
\begin{aligned}
B(v) & =\int_{0}^{\infty} d \beta f(\beta)\left(\frac{\beta m}{2 \pi}\right)^{d / 2} \exp \left[-\frac{\beta m v^{2}}{2}\right] \\
& =\left(\frac{\beta_{0} m}{\pi n}\right)^{d / 2} \frac{\Gamma\left(\frac{n+d}{2}\right)}{\Gamma\left(\frac{n}{2}\right)}\left(1+\frac{\beta_{0}}{n} m v^{2}\right)^{-\frac{n+d}{2}},
\end{aligned}
$$

which can be mapped onto the $q$-Gaussian distribution, emerging within the formalism of nonextensive statistical mechanics (NSM), with an entropic index $q^{\mathrm{NSM}}:=1+2 /(n+$ $d$ ) and an effective inverse temperature $\widetilde{\beta}:=\beta_{0}(n+d) / n$. It can also be mapped onto the family of kappa distributions with the identification $\kappa:=-1+(n+d) / 2$, and $\widetilde{\beta} \equiv \beta_{0}(n+$ $d-2) / 2 n$ for the "traditional" kappa distribution, introduced by Olbert [32] and Vasyliūnas [33], or $\widetilde{\beta}:=\beta_{0}(n+d) / n$ for a slightly different form introduced by Leubner [34] and adopted by many authors $[1,35,36]$. In the statistics literature, distributions in the form of Eq. (3) are known as Student's $t$ distributions and they constitute a particular case of the Burr type III distribution [37].

(b) Inverse- $\chi^{2}$ superstatistics. In this case, instead of $\beta$, the temperature $\left(\beta^{-1}\right)$ itself is $\chi^{2}$ distributed with degree $n$. That is, $f(\beta)$ is given by the inverse- $\chi^{2}$ distribution,

$$
f(\beta)=\frac{\beta_{0}}{\Gamma\left(\frac{n}{2}\right)}\left(\frac{n \beta_{0}}{2}\right)^{n / 2} \beta^{-n / 2-2} e^{-\frac{n \beta_{0}}{2 \beta}} .
$$

The corresponding velocity distribution reads as

$$
\begin{aligned}
B(v)= & \frac{\beta_{0}}{2 \Gamma\left(\frac{n}{2}\right)}\left(\frac{m}{2 \pi}\right)^{d / 2}\left(\frac{\beta_{0} n}{2}\right)^{n / 2}\left(\frac{m v^{2}}{\beta_{0} n}\right)^{\frac{2-d+n}{4}} \\
& \times \mathcal{K}_{\frac{2-d+n}{2}}\left(\sqrt{n m \beta_{0}}|v|\right),
\end{aligned}
$$

where $\mathcal{K}_{\alpha}(x)$ is the modified Bessel function of the second kind.

(c) Lognormal superstatistics. In this case, $\beta$ follows a lognormal distribution,

$$
f(\beta)=\frac{1}{\sqrt{2 \pi} s \beta} \exp \left\{\frac{-\left(\ln \frac{\beta}{\mu}\right)^{2}}{2 s^{2}}\right\},
$$

with an average given by $\beta_{0}=\mu e^{s^{2} / 2}$. In this last case, the velocity distribution $B(v)$ cannot be obtained in closed 

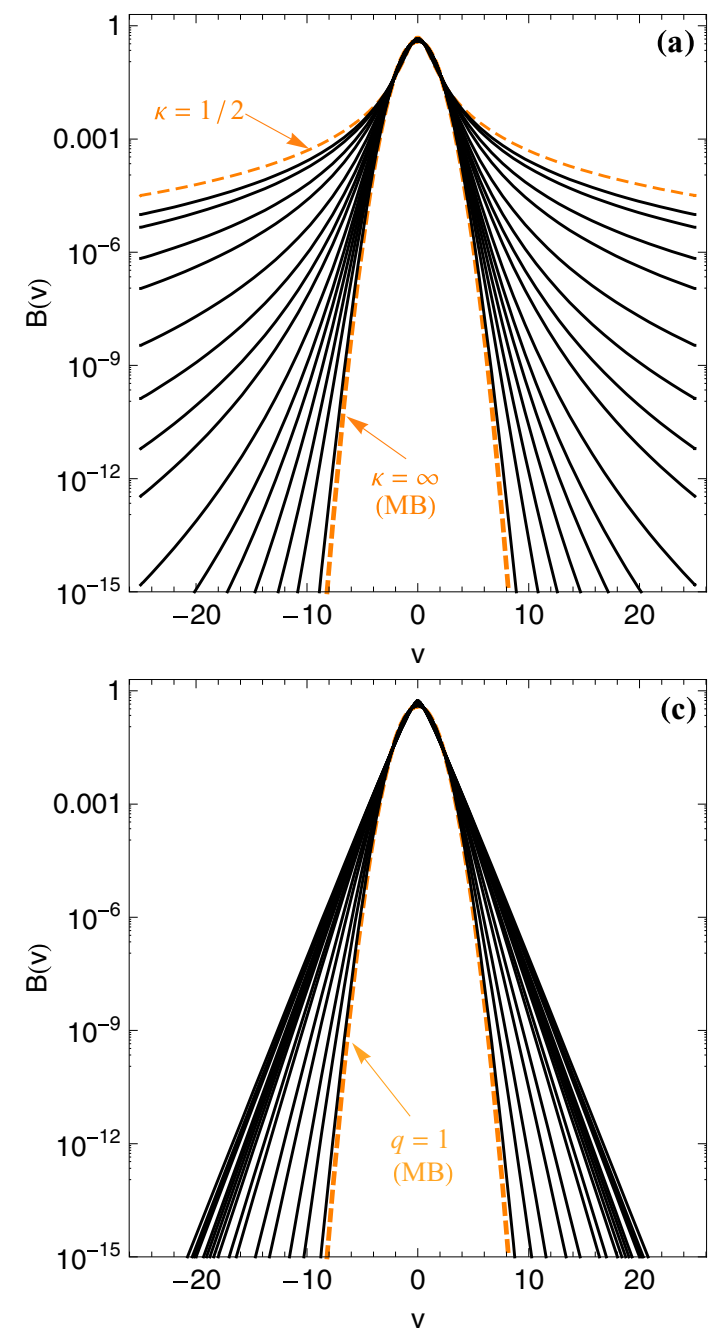
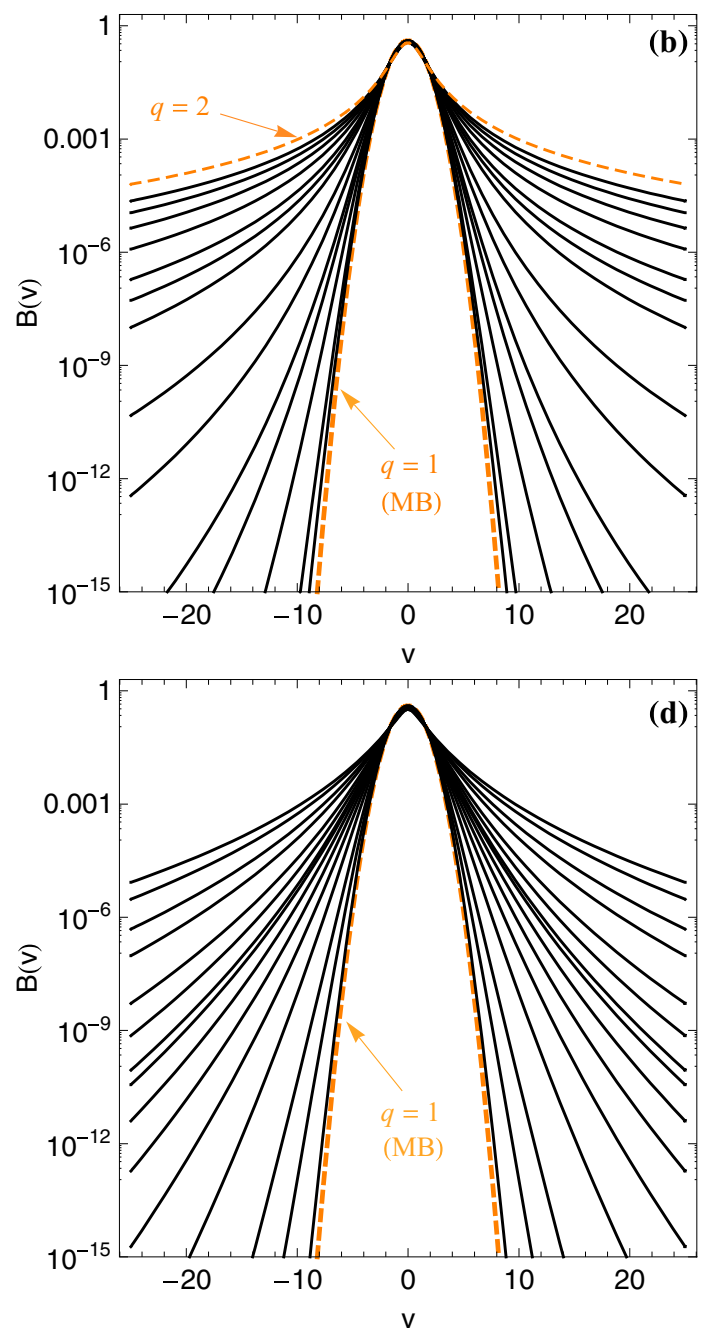

FIG. 1. Examples of kappa distributions (a), $\chi^{2}$ (b), inverse- $\chi^{2}$ (c), and lognormal (d) superstatistics, in the one-dimensional case for $\beta_{0} m=1$. Dashed lines correspond to the limiting cases: the MB distribution $(\kappa=\infty$ or $q=1)$ in one limit, and the critical value beyond which the second moment diverges, i.e., $\kappa=1 / 2$ (a) and $q=2$ (b), in the other. For inverse- $\chi^{2}$ and lognormal superstatistics, the second moment is always finite.

form but can easily be computed numerically. The above distributions, i.e., Eqs. (2), (4), and (6), constitute the three universality classes of superstatistics. Most experimentally relevant situations fall into one of these classes or simple combinations of them [38]. $\chi^{2}$ superstatistics corresponds to the statistics arising from NSM and have been observed in many situations [39]. Experimental evidence of lognormal superstatistics has been found, for instance, in the context of Lagrangian and Eulerian turbulence [38,40,41], and candidate systems for inverse- $\chi^{2}$ superstatistics have been reported in Refs. [17,42]. The velocity distributions arising from these classes cover the main families of distributions encountered in nature: velocity distributions associated with $\chi^{2}$ superstatistics exhibit power-law tails for large $|\mathbf{v}|$, those associated with inverse- $\chi^{2}$ superstatistics exhibit exponential decays, while lognormal superstatistics produces truncated power laws. In this sense, these universality classes constitute the optimal basis set onto which to expand $f(\beta)$ in an inverse problem, i.e., in the process of inferring $f(\beta)$ given some available information about $B(v)$.
Note that for small fluctuations, the three superstatistical distributions can be expanded around the MB distribution as $[12,27]$

$$
B(v) \propto\left(1+\frac{\sigma_{f}^{2} \beta_{0}^{2} m^{2} v^{4}}{8}\right) e^{-\frac{\beta_{0} m v^{2}}{2}},
$$

where $\sigma_{f}^{2}$ is the variance of $f(\beta)$. That is, in this limit the three classes of superstatistics exhibit a universal behavior corresponding to the Cairns nonthermal distribution [31], with an index $\alpha \equiv \sigma_{f}^{2} / 8$. In Fig. 1, we show examples of velocity distributions emerging from the three universality classes of superstatistics, i.e., $\chi^{2}$ [Eq. (3)], inverse $\chi^{2}$ [Eq. (5)], and lognormal (computed numerically), for different degrees of fluctuations [see Eq. (13) below], together with the traditional kappa distribution for comparison. Clearly, inverse- $\chi^{2}$ superstatistics are not suitable for modeling space plasma phenomena since they exhibit exponential decays in $|\boldsymbol{v}|$. From another hand, both $\chi^{2}$ and lognormal superstatistics have profiles very similar to the observed velocity distributions, 
usually fitted with the kappa distribution. One difficulty with the lognormal superstatistics is that the associated velocity distribution cannot be obtained in closed form and has to be treated numerically. Note however that the velocity moments $\left\langle v^{l}\right\rangle$ can be computed analytically, opening up the possibility of a macroscopic (fluidlike) formulation in an exact fashion. Besides, lognormal velocity distributions admit a complete (infinite) set of velocity moments that are well defined for all parameter values, overcoming therefore one major problematic of the kappa distribution [43]. To show this, let us write the superstatistical velocity moments as follows:

$$
\left\langle v^{l}\right\rangle_{f}=\int v^{l} B(v) d^{d} v=\left\langle\left\langle v^{l}\right\rangle_{\mathrm{MB}}\right\rangle_{f(\beta)},
$$

where $\langle\cdot\rangle_{\mathrm{MB}}$ stands for an average over the ( $d$-dimensional) MB velocity distribution and $\langle\cdot\rangle_{f(\beta)}$ an average over the temperature distribution $f(\beta)$. Combining the moments of the three distributions, i.e., Eqs. (2), (4), and (6),

$$
\begin{aligned}
\left\langle\beta^{l}\right\rangle_{\chi^{2}} & =\frac{\Gamma\left(\frac{n}{2}+l\right)}{\Gamma\left(\frac{n}{2}\right)}\left(\frac{2}{n}\right)^{l} \beta_{0}^{l}, \\
\left\langle\beta^{l}\right\rangle_{\text {inv }-\chi^{2}} & =\frac{\Gamma\left(\frac{n}{2}+1-l\right)}{\Gamma\left(\frac{n}{2}\right)}\left(\frac{n}{2}\right)^{l-1} \beta_{0}^{l}, \\
\left\langle\beta^{l}\right\rangle_{\mathrm{LN}} & =e^{l(l-1) s^{2} / 2} \beta_{0}^{l},
\end{aligned}
$$

with the moments of the MB (Gaussian) distribution

$$
\left\langle v^{l}\right\rangle_{\mathrm{MB}}=\frac{(l+d-2) ! !}{(\beta m)^{l / 2}}
$$

( $l$ even), one may obtain all velocity moments in an exact form. In particular, the first-order (velocity) and second-order (stress tensor per unit mass) moments read as

$$
\begin{aligned}
& \mathcal{M}^{1}=\int \mathbf{v} B(v) d^{d} v=\mathbf{0}, \\
& \overleftrightarrow{\mathcal{M}}^{2}=\int \mathbf{v} \otimes \mathbf{v} B(v) d^{d} v .
\end{aligned}
$$

The first-order moment $\mathcal{M}^{1}$ vanishes due to the isotropy of the MB distribution. Assuming that the stress tensor is described by an isotropic pressure so that the dyadic product can be contracted, $\boldsymbol{v} \otimes \boldsymbol{v} \rightarrow v^{2}$, one has for the three superstatistics

$$
\begin{aligned}
\left\langle v^{2}\right\rangle_{\chi^{2}} & =\frac{n}{n-2}\left\langle v^{2}\right\rangle_{\mathrm{MB}} \quad(n>2), \\
\left\langle v^{2}\right\rangle_{\mathrm{inv}-\chi^{2}} & =\frac{n+2}{n}\left\langle v^{2}\right\rangle_{\mathrm{MB}}, \\
\left\langle v^{2}\right\rangle_{\mathrm{LN}} & =e^{s^{2}}\left\langle v^{2}\right\rangle_{\mathrm{MB}} .
\end{aligned}
$$

To draw a comparison between the different classes of fluctuations, it is convenient to define the universal parameter (different from the entropic index $q^{\mathrm{NSM}}$ used in NSM) as $q:=\left\langle\beta^{2}\right\rangle /\langle\beta\rangle^{2}$ that measures the strength of fluctuations. It can be thought of as a "geometric variance" that reduces to 1 in the absence of fluctuations, i.e., when the temperature distribution $f(\beta)$ shrinks to a Dirac delta. For the three universality classes, it can be expressed as

$$
q:=\frac{\left\langle\beta^{2}\right\rangle_{\chi^{2}}}{\beta_{0}^{2}}=1+\frac{2}{n} \quad(n>2)
$$

$$
\begin{aligned}
& q:=\frac{\left\langle\beta^{2}\right\rangle_{\text {inv- }-\chi^{2}}}{\beta_{0}^{2}}=\frac{n}{n-2}, \\
& q:=\frac{\left\langle\beta^{2}\right\rangle_{\mathrm{LN}}}{\beta_{0}^{2}}=e^{s^{2}},
\end{aligned}
$$

from which Eq. (12) can be rewritten in a unified manner as

$$
\left\langle v^{2}\right\rangle_{i}=d \cdot \phi_{i}(q) \frac{T_{0}}{m} \quad(i=1,2,3),
$$

where $T_{0} \equiv \beta_{0}^{-1}$ is the mean temperature and

$$
\begin{aligned}
& \phi_{1}(q) \equiv \frac{1}{2-q} \quad(1<q<2), \\
& \phi_{2}(q) \equiv \frac{2 q-1}{q}, \\
& \phi_{3}(q) \equiv q,
\end{aligned}
$$

with $i=1,2$, and 3 corresponding, respectively, to $\chi^{2}$, inverse- $\chi^{2}$, and lognormal superstatistics. In the limit $q \rightarrow 1$, the distribution $f(\beta)$ approaches a Dirac delta centered at $\beta_{0}$ and $\left\langle v^{2}\right\rangle_{i}$ reduces to the MB second-order moment, i.e., $d \cdot T_{0} / m$. Note that the requirement for having a finite energy (and pressure) necessarily narrows the acceptable parameter range for $\chi^{2}$ superstatistics to $1 \leqslant q<2$. A similar restriction applies to the family of kappa distributions, imposing that $\kappa>$ $d / 2$ [43]. Such a limitation restricts the derivation of a closed system of fluid equations and imposes constraints in fitting observations. Parameter values leading to diverging moments are usually excluded from observational reports [44], although there is some recent indication [45] of parameter values lying outside the allowable range. This motivates recently the introduction of regularized kappa distributions [43] by adding an extra parameter that acts as a cutoff at high velocities. Interestingly, velocity distributions corresponding to lognormal superstatistics do not suffer from this drawback and remain valid in fitting data for high values of $q$. A word of caution is however in order; likewise for all heavy-tailed distributions, lognormal velocity distributions are not immune from possible unphysical character related to superluminal particles. In fact, since all moments are obtained through integration over the velocity space which, in the nonrelativistic treatment, extends to infinity, the presence of heavy tails may lead to a significant contribution from particles with superluminal velocities, which necessitates truncating their contribution.

Before closing this section, it is worth noting that we are tacitly adopting here a type-B formulation of superstatistics since we are considering locally normalized $\mathrm{MB}$ distributions that are averaged over $f(\beta)$ [see for instance Eq. (3)]. The other alternative, known as type-A superstatistics [12], consists of working with unnormalized canonical distributions and normalize the marginal distribution at the end of the process. One may, however, easily switch from type-B superstatistics to type-A superstatistics, and vice versa, by properly redefining the distribution $f(\beta)$. Since we are adopting type-B superstatistics, the parameter $q$ used here [Eq. (13)], in the case of $\chi^{2}$ superstatistics, is different from the one used in NSM. The latter is obtained within the transformation $q^{\mathrm{NSM}}:=1+[2(q-1)] /[2+d(q-1)]$, and the restriction for having a finite second-order moment implies $q^{\mathrm{NSM}}<5 / 3$. 


\section{INDIRECT OBSERVATION: DISPERSION RELATIONS}

When direct in situ measurements of the distribution function are not available, one may seek the signature of particular distributions in a number of physical processes. In this case, noncanonical velocity distributions are not directly observed but postulated in order to account for indirect measurements. A meaningful example is that of the Cairns nonthermal distribution [31] that was introduced ad hoc to account for the observation of solitary electrostatic structures involving density depletions in the upper ionosphere. Among measurements that have led to the consideration of noncanonical distributions are those concerned with plasma oscillations. In this case, experiments provide dispersion relations that deviate from what one would expect in the case of the MB distribution. One particularly studied example is the set of data provided by Van Hoven [46] in the 1960s that has been argued to be a manifestation of nonextensivity [47] and subsequently used to constrain the entropic index [48]. Here we wish to show that such an effect on plasma oscillations is not associated with the particular case of Tsallis statistics but is a general feature of nonequilibrium stationary distributions. To show this, we consider a field-free plasma, of uniform density $n$, which is not initially in an equilibrium state but in a stationary nonequilibrium state, described by a distribution $B(v)=\left\langle f_{\mathrm{MB}}(v)\right\rangle_{f}$ [cf. Eq. (1)], where $f_{\mathrm{MB}}(v)$ is the $\mathrm{MB}$ distribution. If at a given time $t$, a small amount of charge is displaced in the plasma, the distribution function is perturbed accordingly,

$$
f(\mathbf{r}, \mathbf{v} ; t)=B(v)+\delta f(\mathbf{r}, \mathbf{v} ; t) .
$$

For time intervals shorter than the binary collision time $\tau$, the distribution $f(\mathbf{r}, \mathbf{v} ; t)$ obeys the collisionless Boltzmann (Vlasov) equation

$$
\frac{\partial f(\mathbf{r}, \mathbf{v} ; t)}{\partial t}+\mathbf{v} \cdot \frac{\partial f(\mathbf{r}, \mathbf{v} ; t)}{\partial \mathbf{r}}+\frac{e \boldsymbol{\nabla} \phi}{m} \cdot \frac{\partial f(\mathbf{r}, \mathbf{v} ; t)}{\partial \mathbf{v}}=0,
$$

where $e$ is the elementary charge and $\phi$ the electrostatic potential produced by the perturbation. For small perturbations ( $\delta f \ll B$ ), one may linearize Eq. (17) and couple it with the Poisson equation to form a closed set of equations as follows:

$$
\begin{aligned}
& \frac{\partial \delta f}{\partial t}+\mathbf{v} \cdot \frac{\partial \delta f}{\partial \mathbf{r}}+\frac{e}{m} \nabla \phi \cdot \frac{\partial B}{\partial \mathbf{v}}=0, \\
& \nabla^{2} \phi=-4 \pi e n \int \delta f d^{3} v .
\end{aligned}
$$

The above set of equations may be solved simultaneously, following the line of the "Landau school" [49], by using standard integral transformation techniques, or equivalently by performing a decomposition in Fourier modes. That is,

$$
\delta f \sim e^{i(\mathbf{k} \cdot \mathbf{r}-\omega t)} \quad \text { and } \quad \phi \sim e^{i(\mathbf{k} \cdot \mathbf{r}-\omega t)} .
$$

We consider here, without loss of generality, the $x$ axis to be along the direction of the wave vector $\mathbf{k}$ and let $v \equiv v_{x}$. Equation (18) leads to

$$
D(k, \omega):=1-\frac{4 \pi e^{2}}{k^{2} m} \int \frac{\partial B / \partial v}{v-\omega / k} d^{3} v=0 .
$$

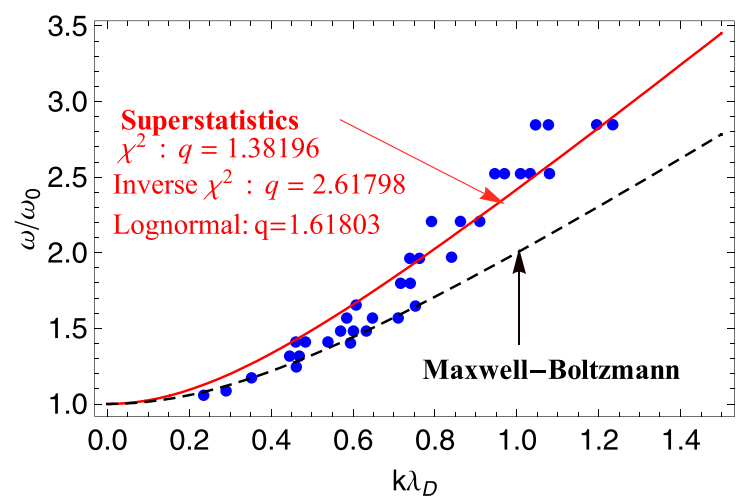

FIG. 2. Circles correspond to the experimental data set of Van Hoven [46]. The dashed line represents the Bohm-Gross relation, arising from the MB distribution, and the solid line the best fit using Eq. (22), corresponding to $\omega / \omega_{0}=\sqrt{1+4.85408\left(k \lambda_{D}\right)^{2}}$.

For $v \ll \omega / k$, one may Taylor-expand the integrand of Eq. (20) in powers of $k$. Bearing in mind that

$$
\frac{\partial B}{\partial v}=\frac{\partial\left\langle f_{\mathrm{MB}}\right\rangle_{f}}{\partial v}=\left\langle\frac{\partial f_{\mathrm{MB}}}{\partial v}\right\rangle_{f}
$$

one obtains the following dispersion relation

$$
\frac{\omega^{2}}{\omega_{0}^{2}}=1+3 \phi_{i}(q)\left(\lambda_{D} k\right)^{2}
$$

where $\omega_{0} \equiv\left(4 \pi n e^{2} / m\right)^{1 / 2}$ is the natural oscillation plasma frequency and $\lambda_{D} \equiv\left(T_{0} / 4 \pi n e^{2}\right)^{1 / 2}$ is the Debye screening length defined at the mean temperature $T_{0}$, while the correction terms $\phi_{i}(q)(i=1,2,3)$, due to temperature fluctuations, are given in Eq. (15) for the three superstatistics. In the absence of fluctuations, one has $\phi_{i}(1)=1$, and Eq. (22) reduces to the standard Bohm-Gross relation [50]. In Fig. 2, the dispersion relation (22) is confronted by the experimental data set of Van Hoven [46]. One may see that the three classes of fluctuations have qualitatively the same effect on the dispersion relation that tends to show a better agreement with experimental data. From the measurements, one may estimate the strength of fluctuations, i.e., $q=\langle\beta\rangle / \beta_{0}^{2}$, for each class, by curve fitting. For this particular data set, the estimated values of $q$ are shown in Fig. 2.

Note that, at this order of approximation, the form of the dispersion relation is independent of the precise form of the velocity distribution. Equation (22) has the same form as the standard Bohm-Gross relation while the only difference appears in the factor associated with the thermal motion. As a consequence, it can only fit the data points that depart the most from the standard expression (those in the large- $k$ region) at the expense of data points in the low- $k$ region. To achieve a better agreement with measurements, one has to go beyond the idealized linear dispersion and consider extensions of the dispersion relation accounting for other effects that may come into play, such as the effects of a finite geometry or plasma nonuniformity (see for instance [51]). Such dispersion relations are however quite challenging and in general do not allow for an analytic investigation. 

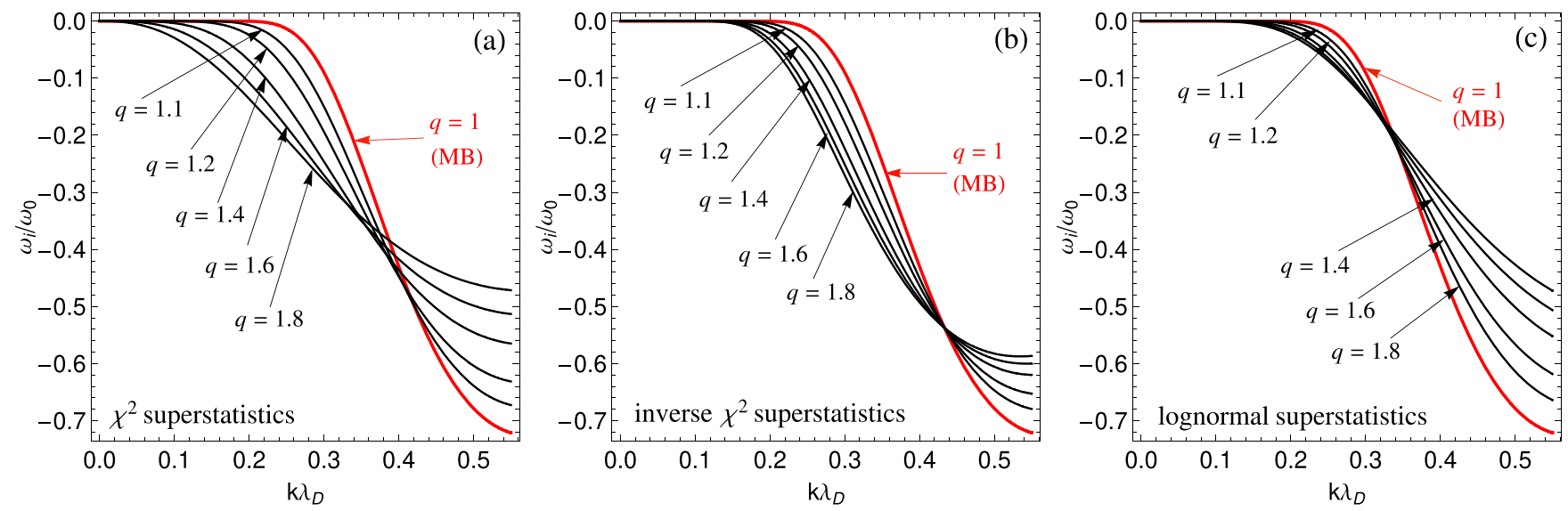

FIG. 3. The damping rate as a function of the wave number for (a) $\chi^{2}$ [Eq. (25)], (b) inverse- $\chi^{2}$ [Eq. (26)], and (c) lognormal superstatistics [Eq. (27), computed numerically], for different values of $q:=\left\langle\beta^{2}\right\rangle / \beta_{0}^{2}$.

Note that due to the singularity in velocity space, the integral (20) is not properly defined. This singularity induces an imaginary part in the dispersion relation, responsible for the Landau damping. It is worth studying the effect of fluctuations on this process. We restrict ourselves to the case of weak damping (the imaginary part $\omega_{i}$ is considered much smaller than the real part $\omega_{r}$ ) which remains tractable analytically for the first two classes of superstatistics. By making the analytic continuation of the integral over $v$, along the real axis, which passes under the pole at $v=\omega / k$, one may explicitly find the real and imaginary parts of the dielectric function (20) as follows:

$$
\begin{aligned}
& D_{r}\left(k, \omega_{r}\right)=1-\frac{4 \pi n e^{2}}{m k^{2}} \mathrm{PV} \int \frac{\partial B(v) / \partial v}{v-\frac{\omega_{r}}{k}} d v, \\
& D_{i}\left(k, \omega_{r}\right)=-\pi\left(\frac{4 \pi n e^{2}}{m k^{2}}\right)\left[\frac{\partial}{\partial v} B(v)\right]_{v=\frac{\omega r}{k}},
\end{aligned}
$$

where PV $\int$ denotes the Cauchy principal value. By neglecting second-order terms in $\omega_{i} / \omega_{r}$, one may obtain the imaginary part $\omega_{i}$ from the relation [50]

$$
\omega_{i}=-\frac{D_{i}\left(k, \omega_{r}\right)}{\partial D_{r}\left(k, \omega_{r}\right) / \partial \omega_{r}} .
$$

Following these standard lines, one may compute $\omega_{i}$ for the different universality classes of superstatistics. For $\chi^{2}$ and inverse- $\chi^{2}$ superstatistics, we obtain closed form expressions as follows:

$$
\begin{gathered}
\omega_{i}=-\sqrt{\frac{\pi}{8}} \frac{\Gamma\left[\frac{3}{2}+\frac{1}{q-1}\right]}{\Gamma\left[\frac{1}{q-1}\right]} \frac{(q-1)^{3 / 2} \omega_{0}}{\left(\lambda_{D} k\right)^{3}}\left[1+\frac{(q-1)}{2 \lambda_{D}^{2} k^{2}}\right]^{\frac{1}{1-q}-\frac{1}{2}} \\
\omega_{i}=-\sqrt{\frac{\pi}{8}} \frac{2^{\frac{q-3}{4(q-1)}+\frac{1}{2}}}{\Gamma\left[\frac{q}{q-1}\right]}\left(\frac{q}{q-1}\right)^{\frac{q}{q-1}}\left[\frac{q-1}{q \lambda_{D}^{2} k^{2}}\right]^{\frac{1+q}{4(q-1)}} \\
\times \frac{\omega_{0}}{\lambda_{D}^{3} k^{3}} \mathcal{K}_{\frac{q+1}{2(q-1)}}\left(\sqrt{\frac{2 q}{(q-1) \lambda_{D}^{2} k^{2}}}\right)
\end{gathered}
$$

where $\mathcal{K}_{\alpha}(x)$ is the modified Bessel function of the second kind. For lognormal superstatistics, one has

$$
\omega_{i}=-\sqrt{\frac{\pi}{8}} \int_{0}^{\infty} d \beta \frac{(\beta m)^{3 / 2} \omega_{0}^{4}}{\sqrt{2 \pi \ln (q)} \beta k^{3}} e^{-\frac{[\ln (\beta / \sqrt{ })]^{2}}{2 \ln (q)}}-\frac{\beta m \omega_{0}^{2}}{2 k^{2}},
$$

for which there is no closed-form solution, so it will be treated numerically. One may easily check that Eqs. (25)-(27) reduce to the standard Landau result [50]

$$
\omega_{i}=-\sqrt{\frac{\pi}{8}} \frac{\omega_{0}}{\left(k \lambda_{D}\right)^{3}} e^{-\frac{1}{2\left(k \lambda_{D}\right)^{2}}}
$$

in the limit of vanishing fluctuations, that is, for $q \rightarrow 1$ or equivalently $f(\beta) \rightarrow \delta\left(\beta-\beta_{0}\right)$.

Figure 3 shows the damping rate $\omega_{i} / \omega_{0}$ as a function of the wave number for the three universality classes, with different values of $q:=\left\langle\beta^{2}\right\rangle / \beta_{0}^{2}$. Here again, one may note that the effect of the different classes of fluctuations is qualitatively the same. For the three universality classes, the damping rate shows two different stages: for wave numbers smaller than a ( $q$-dependent) critical value, the damping increases due to fluctuations, while for bigger wave numbers, the damping decreases for larger values of $q$. This is similar to the effect predicted for a nonextensive plasma $[47,52]$ in the suprathermal regime, that is, for Tsallis statistics without thermal cutoff. Similar behavior was also reported recently [53] in the case of Kaniadakis distributions [54]. It is worth observing that for all superstatistics, one always has $\omega_{i}<0$, giving rise to damped modes, while growing (unstable) modes do not appear. This is because the velocity distributions emerging from superstatistics, being merely a superposition of MB distributions at different temperatures, are single humped, viz., the Gardner theorem. This implies that there are always more particles having velocities slightly less than the phase velocity $\omega / k$, hence gaining energy from the wave, than particles having velocities slightly greater, hence losing energy to the wave.

Before closing this section, it is worth highlighting the generality of the present approach. In fact, although we are mainly concerned here with plasma excitations, the same picture is applicable in other contexts. One may think for instance of hybrid-phonon modes, Bogoliubov excitations in dipolar condensates, or the Jeans instability of self-gravitating 
matter. In this last example, the superstatistical generalization may be straightforwardly addressed, by virtue of the formal analogy between the Jeans analysis and plasma oscillations. By coupling the Vlasov equation with the Poisson equation for the gravitational field, and making use of the so-called "Jeans swindle," one may arrive at the gravitational counterpart of the dispersion relation (22) as

$$
\omega^{2}=-\omega_{J}^{2}+k^{2}\left\langle v^{2}\right\rangle_{i},
$$

where $\omega_{J} \equiv(4 \pi G m n)^{1 / 2}$ is the Jeans frequency. Equation (29) indicates that the Jeans instability can be saturated by thermal effects associated with a finite value of the mean-squared velocity $\left\langle v^{2}\right\rangle_{i}$ of the self-gravitating matter. As thermal fluctuations tend to increase the second-order velocity moment [see Eq. (14)], they tend to stabilize the self-gravitating instability for smaller values of $k$, or larger wavelengths. More precisely, the Jeans wavelength, above which gravitational instability occurs, can be deduced from Eq. (29) as

$$
\tilde{\lambda_{J}}=\sqrt{\phi_{i}(q)} \lambda_{J}, \quad \lambda_{J} \equiv \sqrt{\frac{\pi T_{0}}{G m^{2} n}},
$$

where $\lambda_{J}$ is the Jeans wavelength in the absence of fluctuations. Here again, in the particular case of $\chi^{2}$ superstatistics, the whole picture reduces (with a different notation) to the Jeans analysis in the context of NSM, in the suprathermal regime, addressed by many authors from the kinetic [55] or the hydrodynamic [56,57] point of view.

\section{DIRECT OBSERVATION: ULYSSES ELECTRON DISTRIBUTIONS}

When direct in situ measurements of the distribution function are not available, it is not surprising that different distributions may lead to the same effect on observable quantities, but does this also hold true when direct observations of the distribution functions are involved? Here again, it is not ensured that one can distinguish between different distributions. One may find examples within the kappa distribution itself: The original distribution introduced by Olbert [32] and Vasyliūnas [33] slightly differs from the one introduced by Leubner [34]. Further, even by adopting one or the other, yet two slightly different distributions arise depending on whether one identifies the width of the distribution with a "fundamental" temperature that—by definition—should not depend on $\kappa$ [58] or, alternatively, associate it with the temperature in the kinetic sense (that is related to the second-order velocity moment $\left.\left\langle v^{2}\right\rangle\right)$ and therefore does depend on $\kappa$ [59]. These two alternatives are known in the literature as kappa $\mathrm{A}$ and kappa B (see for instance [59-61] for an elaborate discussion). An important consequence of these two possible representations of the kappa distribution appears in defining the proper Maxwellian limit. As pointed out in Ref. [59], when the kappa distribution is used to fit a set of measurements, depending on its interpretation as kappa A or kappa B, two Maxwellian limits, with different temperatures, may arise. The superstatistical picture dispels such an ambiguity by regarding such distributions as a manifestation of a nonequilibrium situation. In a nonequilibrium state, attributing a single temperature to the whole system is elusive. This is even more so given that the (inverse) temperature $\beta$ is not an observable in such a scenario, as demonstrated in $[30,62]$. Nevertheless, the formalism of superstatistics draws a clear distinction between the mean temperature $T_{0}$, which characterizes the MB distribution in the limit of vanishing fluctuations, i.e., $q \rightarrow 1$, and the kinetic temperature (proportional to $\left\langle v^{2}\right\rangle$ ) that depends on both the class of fluctuations and their strength [cf. Eqs. (14) and (15)].

Here, we confront the velocity distributions emerging from superstatistics with typical observations of non-Gaussian velocity distributions in a collisionless plasma. We ignore the class of inverse- $\chi^{2}$ superstatistics since the velocity distributions, in this case, clearly depart from typical observations [see Fig. 1(c)]. We will only consider velocity distributions emerging from $\chi^{2}$ superstatistics (2) and lognormal superstatistics (6).

More precisely, we use observed $3 d$ electron velocity distributions obtained with the solar wind electron plasma instrument on board the Ulysses spacecraft [63]. This instrument measures velocity distributions of electrons with central energies ranging from $0.86 \mathrm{eV}$ to $814 \mathrm{eV}$, which are known to be well fitted with the kappa distribution [64].

To fit with the observations, the superstatistical velocity distributions are renormalized to the density, that is, $B(v) \rightarrow$ $n_{e} B(v)$, where $n_{e}$ is fixed as the total electron density obtained from observations. Fixing the temperature is less straightforward: One might be tempted to identify the observed temperature with the mean temperature $T_{0}$. Indeed, in an in situ experiment, if the temperature is measured in an event-byevent analysis, one would expect that the average of measurements would converge to $T_{0}$. In space plasmas, however, the temperature is often measured indirectly by analyzing the observed velocity distributions; it is obtained by integrating the observed electron distributions over the whole velocity range (see for instance Ref. [64]). In this case, the temperature $T_{\mathrm{obs}}$, deduced from the observed velocity distributions, is affected by fluctuations and must be taken as an effective temperature. It is proportional to the second-order velocity moment $\left\langle v^{2}\right\rangle_{i}$ and hence depends on $q$. Using Eq. (14), one may link the effective temperature $T_{\mathrm{obs}}$ and the mean temperature $T_{0}$ as

$$
T_{\mathrm{obs}}=\phi_{i}(q) T_{0} \Leftrightarrow T_{0}=\phi_{i}(q)^{-1} T_{\mathrm{obs}} \quad(i=1,2,3)
$$

with $\phi_{i}(q)(i=1,2,3)$ given in Eq. (15) for the three superstatistics.

After rewriting the $\chi^{2}$ [Eq. (2)] and the lognormal [Eq. (6)] distributions in terms of $T_{\mathrm{obs}}$ and $q$, we use the associated velocity distributions to fit typical Ulysses electron distributions. Results are shown in Fig. 4 where $\ln [B(v)]$ is plotted to better characterize the importance of the tails. In Table I, we give the values of $q$ that produce the best fits together with the corresponding normalized root-mean-square error (RMSE), defined as

$$
\mathrm{RMSE}:=\sqrt{\frac{1}{n} \sum_{i}\left(\frac{y_{i}^{(q)}-y_{i}}{y_{i}}\right)^{2}},
$$

where $y_{i}^{(q)}$ are the predicted values of $\ln [B(v)]$ for the two superstatistical models and $y_{i}$ the measured values. One may see that both superstatistics reproduce quite well the observational data. In particular for low densities, the lognor- 

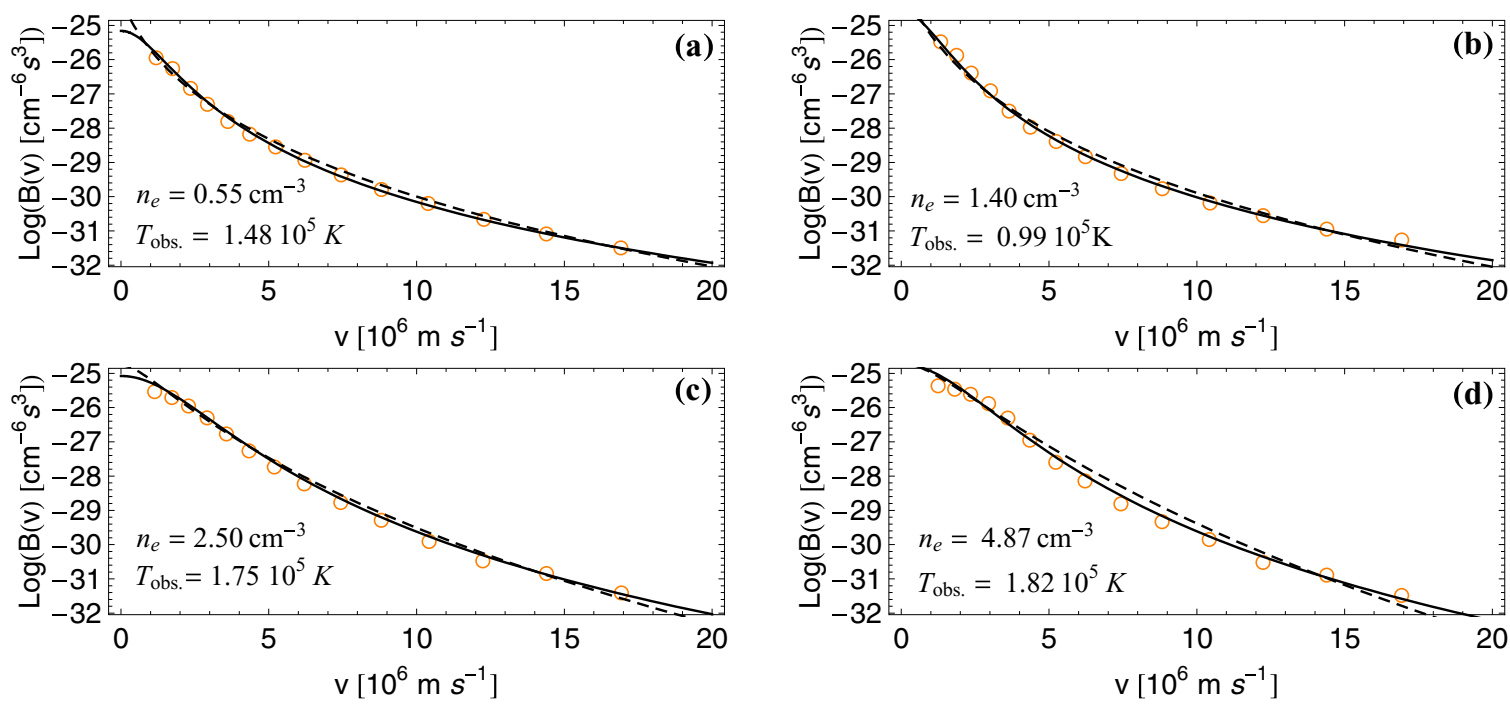

FIG. 4. Four typical Ulysses electron distributions. The open circles represent averages of the observed electron distributions over all spatial angles from Ref. [64]. The solid line corresponds to the best fit obtained with $\chi^{2}$ superstatistics [Eq. (3)] and the dashed line represents the best fit with lognormal superstatistics (treated numerically). Parameter values and mean-squared errors are given in Table I.

mal superstatistics nicely fit the data and even outperform the $\chi^{2}$ model in the lowest density case (a).

\section{THERMODYNAMIC ASPECTS}

Let us discuss here some thermodynamic aspects that emerge within the superstatistical picture, and show how the paradigm clarifies those aspects. The first aspect to be considered is the heat capacity. A possible link between the heat capacity and noncanonical distributions can be traced back to some textbooks [65] where power-law distributions appear in an intermediate step in the derivation of the canonical distribution, the latter being obtained by letting the system size go to infinity. Quite naturally, early attempts [66] at explaining Tsallis statistics have associated the entropic index $q$ to the heat capacity.

The underlying idea is that both the canonical and the microcanonical ensembles, in equilibrium statistical mechanics, represent ideal cases: the canonical ensemble applies to the case of a system in contact with an infinite heat bath (the temperature is fixed and the energy may fluctuate) and the microcanonical ensemble applies to the case of an isolated system (the energy is fixed and the temperature fluctuates). Between these two extreme cases lie more realistic cases of physical systems in contact with finite heat baths, where both energy and temperature may fluctuate. For those intermediate cases, one may consider the Lindhard equation [67] which is supposed to remain valid all the way from the canonical ensemble to the microcanonical one,

$$
\operatorname{Var}(U)+C_{V}^{2} \operatorname{Var}(T)=T_{0}^{2} C_{V},
$$

where $U$ is the energy and $C_{V}$ is the heat capacity under constant volume. In the canonical ensemble, one has $\operatorname{Var}(T)=0$ (the temperature is fixed) and the system's heat capacity can be expressed in terms of energy fluctuations as

$$
C_{V}=\frac{\operatorname{Var}(U)}{T_{0}^{2}}
$$

while for an isolated system, the energy is fixed $\operatorname{Var}(U)=0$ and the heat capacity can be computed through temperature fluctuations (see for instance Landau and Lifschitz [68]):

$$
\frac{\left\langle\left(T-T_{0}\right)^{2}\right\rangle}{T_{0}^{2}}=\frac{1}{C_{v}} .
$$

For intermediate cases, one may write [69]

$$
\operatorname{Var}(U)=T_{0}^{2} C_{V} \xi, \quad \xi \in(0,1),
$$

where $\xi$ depends on the size of the system. $\xi=0$ corresponds to the case of an isolated system and $\xi=1$ to a system in contact with an infinite heat bath, i.e., canonical ensemble.

TABLE I. Values of $q:=\left\langle\beta^{2}\right\rangle /\langle\beta\rangle^{2}$ for the two universality classes of superstatistics corresponding to observations for high and low speed streams.

\begin{tabular}{lllll}
\hline \hline$f(\beta)$ & \multicolumn{1}{c}{ (a) } & \multicolumn{1}{c}{ (b) } & \multicolumn{1}{c}{ (c) } \\
\hline$\chi^{2}$ & $q \sim 1.674$ & $q \sim 1.633$ & $q \sim 1.371$ & $q \sim 1.328$ \\
& RMSE: $0.47 \%$ & RMSE: $0.38 \%$ & RMSE: $0.29 \%$ & RMSE: $0.54 \%$ \\
lognormal & $q \sim 7.010$ & $q \sim 6.828$ & $q \sim 2.266$ & $q \sim 1.761$ \\
& RMSE: $0.32 \%$ & RMSE: $0.59 \%$ & RMSE: $0.51 \%$ & RMSE: $0.92 \%$ \\
\hline \hline
\end{tabular}


Using Eqs. (33) and (36), one has

$$
\frac{\left\langle\left(T-T_{0}\right)^{2}\right\rangle}{T_{0}^{2}}=\frac{1-\xi}{C_{v}} .
$$

Changing the variable from $T$ to $\beta$,

$$
\frac{\left\langle\left(T-T_{0}\right)^{2}\right\rangle}{T_{0}^{2}}=\frac{\beta_{0}^{2}-\left\langle\beta^{2}\right\rangle}{\left\langle\beta^{2}\right\rangle}=\frac{\left(\frac{\beta_{0}^{2}}{\left\langle\beta^{2}\right\rangle}\right)^{2}\left\langle\beta^{2}\right\rangle-\beta_{0}^{2}}{\beta_{0}^{2}},
$$

and focusing on the case of small fluctuations, one may write for the three superstatistics

$$
\begin{aligned}
\left(\frac{\beta_{0}^{2}}{\left\langle\beta^{2}\right\rangle_{\chi^{2}}}\right)^{2} & =\left(\frac{1}{1+2 / n}\right)^{2} \underset{n \text { large }}{\simeq} 1-4 / n, \\
\left(\frac{\beta_{0}^{2}}{\left\langle\beta^{2}\right\rangle_{\text {inv- } \chi^{2}}}\right)^{2} & =\left(\frac{n-2}{n}\right)^{2} \underset{n \text { large }}{\simeq} 1+4 / n, \\
\left(\frac{\beta_{0}^{2}}{\left\langle\beta^{2}\right\rangle_{\mathrm{LN}}}\right)^{2} & =\left(\frac{1}{e^{s^{2}}}\right)^{2} \underset{s \text { small }}{\simeq} 1-2 s^{2},
\end{aligned}
$$

where, in the last step, we considered the limit of small fluctuations, i.e., $q:=\left\langle\beta^{2}\right\rangle / \beta_{0}^{2}$ close to 1 . In this limit, one has

$$
\frac{\left\langle\left(T-T_{0}\right)^{2}\right\rangle}{T_{0}^{2}} \simeq \frac{\left\langle\beta^{2}\right\rangle-\beta_{0}^{2}}{\beta_{0}^{2}},
$$

and the heat capacity can be estimated as

$$
C_{v}=\frac{1-\xi}{q-1} .
$$

That is, once interpreted as emerging from fluctuations, the observation of non-Maxwellian velocity distributions may provide an estimate of the heat capacity of space plasmas, opening up new prospects for investigating their thermodynamic properties.

Note that although widely used in the literature to estimate the heat capacity from fluctuations, the above relations and the nature of fluctuations have been the subject of controversy. In particular, Eq. (35) has received different interpretations, including the assertion that it is meaningless [70] or a mere formality [71]. We note in this regard recent molecular dynamics simulations [72] that validate these relations in a context similar to ours, that is, for a quasiequilibrium system composed of small subsystems that remain infinitely close to equilibrium.

Other thermodynamic aspects that gain clarity in the superstatistical picture are those related to entropy considerations. In this paradigm, the noncanonical distributions are not constructed from a generalized (nonextensive) entropy functional, as in the case of NSM [39], and the entropy preserves its extensivity. To show this, let us consider the general definition of entropy, valid for both equilibrium and nonequilibrium systems,

$$
S=-\iint f[\ln (f)-1] d^{3} r d^{3} v-N \ln \left(\frac{h^{3}}{m^{3}}\right),
$$

where $f \equiv n B(v)$ is the phase space distribution function normalized to the density $n$, and $h$ is the Planck constant. Equation (42) was originally introduced by Gibbs [73] and (re)discussed more recently in the context of plasma physics

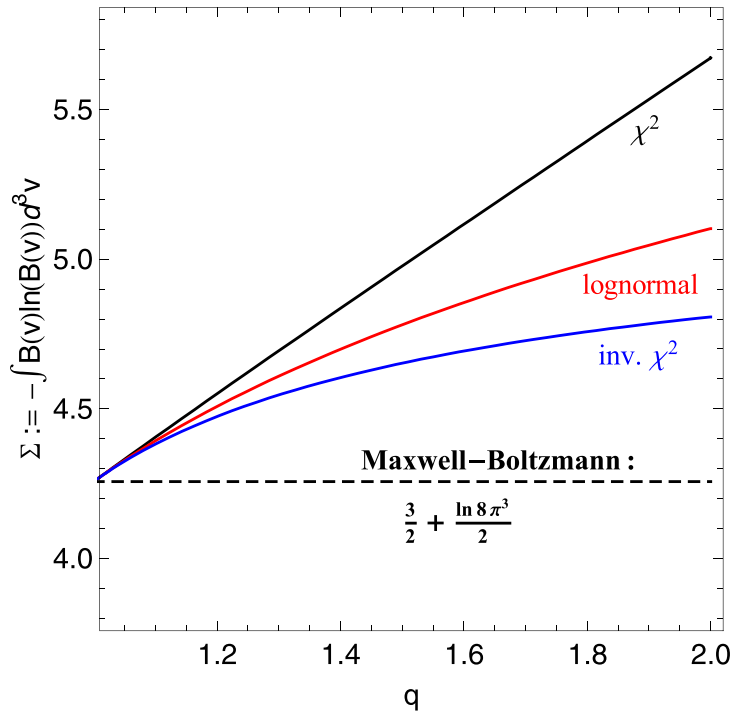

FIG. 5. The scaled entropy $\Sigma$ as a function of $q:=\left\langle\beta^{2}\right\rangle /\langle\beta\rangle^{2}$ for the three universality classes of superstatistics, with $\beta_{0} m=1$.

$[4,74]$. It accounts for the quantum mechanical lower limit of the phase space volume occupied by a single particle and contains the Gibbs factor, avoiding therefore the Gibbs paradox associated with particles' indistinguishability.

Assuming a homogeneous density, one has

$$
\begin{aligned}
S= & -\ln (n) \iint f(v) d^{3} r d^{3} v \\
& -\iint f(v) \ln [B(v)] d^{3} r d^{3} v \\
& +\iint f(v) d^{3} r d^{3} v-N \ln \left(\frac{h^{3}}{m^{3}}\right) .
\end{aligned}
$$

Using the normalization condition

$$
\iint f(v) d^{3} r d^{3} v=n \iint B(v) d^{3} r d^{3} v=N,
$$

Eq. (43) simplifies to

$$
S=N\left[1+\ln \left(\frac{m^{3}}{n h^{3}}\right)+\Sigma\right],
$$

where $\Sigma$ is the "scaled entropy," defined as

$$
\Sigma:=-\int B(v) \ln [B(v)] d^{3} v .
$$

Equation (45) is a generalization of the Sackur-Tetrode entropy in the presence of fluctuations. In the case of vanishing fluctuations, the scaled entropy becomes

$$
\lim _{q \rightarrow 1} \Sigma=\frac{1}{2}\left[3+\ln \left(\frac{8 \pi^{3} T_{0}^{3}}{m^{3}}\right)\right],
$$

and Eq. (45) reduces to the Sackur-Tetrode entropy

$$
S=-N \ln \left(\frac{n h^{3}}{\left(2 \pi m T_{0}\right)^{3 / 2}}\right)+\frac{5}{2} N .
$$

One may see, in light of Eq. (45), that the entropy extensivity $\left(\lim _{N \rightarrow \infty} S / N \neq \infty\right)$ is preserved in the superstatistics scenario, as long as $\Sigma \neq \infty$. Figure 5 shows the effect introduced 
by fluctuations on $\Sigma$, for the three universality classes of superstatistics. Clearly, fluctuations appear to increase the scaled entropy $\Sigma$, and therefore the total entropy (45). That is, fluctuations tend to increase the uncertainty in the velocity space. This is the result one would expect from the discussion in Sec. II, where fluctuations are shown to increase the second-order velocity moments [Eq. (12)]. As one compares between the three universality classes, one may observe that, for the same $q$, the class of $\chi^{2}$ superstatistics is the one that increases the most the second-order moment, while the inverse- $\chi^{2}$ superstatistics class has less effect on $\left\langle v^{2}\right\rangle$. The same "hierarchy" of the three universality classes is recovered by looking into the scaled entropy $\Sigma$.

\section{CONCLUSIONS}

In the present analysis we discuss the possibility of explaining the variety of noncanonical distributions observed in space plasmas, within a general framework, relying solely on statistical arguments. The central concept in this analysis is the paradigm of superstatistics that allows explaining the emergence of a large diversity of anomalous distributions as a consequence of fluctuations, with three possible universal statistical origins. The present approach generalizes our previous effort in this direction [27] and is motivated by the recent proof by Davis et al. [29] that the distributions characterizing collisionless steady-state plasmas necessarily follow superstatistics.

We discuss the two possible scenarios: the case where noncanonical distributions are postulated a priori to account for indirect measurements and the case where a direct measurement of the distributions is available. In this former case, it is pointed out that deviations from canonical distributions found in dispersion relations can be reproduced by the three universality classes of superstatistics, contrary to previous claims $[47,48]$ that they constitute a signature of the particular class of Tsallis statistics. By adopting the paradigm of superstatistics, the nonextensivity is not postulated a priori. Rather, the mechanism is explained by the presence of fluctuations that are known to be present in such experiments, and the extra parameter used to fit the data is no longer a free parameter but is connected to temperature fluctuations. In the case when direct measurements are available, it is shown that, in addition to the class of $\chi^{2}$ superstatistics that is known to correctly reproduce typical observations, the class of lognormal superstatistics also shows similar profiles and constitute a promising alternative, permitting us to overcome limitations of the standard distributions used in the literature, associated with diverging moments [43].

It is instructive to note that the issue addressed here represents a particular case of inverse problem, that is, to construct the distribution $f(\beta)$ characterizing temperature fluctuations, given some experimental knowledge about the velocity distribution $B(v)$. Inverse problems are known to be ill conditioned, meaning that a small deviation in $B(v)$ may lead to large uncertainties in the assessment of $f(\beta)$. In order to reduce this issue, one usually resorts to regularization. In our case, $f(\beta)$ is constrained within a set of candidate distributions corresponding to the three universality classes of superstatistics. In the scenario where a direct measurement of the velocity distributions is available, one can in principle construct the temperature distribution. Indirect measurements, however, such as those associated with dispersion relations, are not sensible to the precise form of the distribution function but depend only on its first moments. In this case, one can no longer construct the full temperature distributions, and the three universality classes yield qualitatively the same effect [see Eq. (22)].

It is shown that the superstatistical picture clarifies the ambiguity of defining the proper Maxwellian limit and offers new perspectives for investigating thermodynamic properties of space plasmas, by virtue of the close relationship between the temperature fluctuations and the heat capacity. Furthermore, the extensivity of the entropy is preserved, overcoming therefore possible inconsistencies [75] in considering generalized (nonextensive) entropy functionals.

The present analysis may open up new prospects for future investigations. In particular, the class of lognormal distributions, which has been ignored so far in the plasma physics literature, may find many applications since it is able to potentially reproduce a large range of observations. As shown here, although the distribution has to be treated numerically, the corresponding velocity moments are well defined and accessible in closed form, opening up the possibility of implementing lognormal superstatistics in fluidlike models. Furthermore, our discussion related to plasma oscillations and Landau damping is very general and remains applicable for investigating the effect of fluctuations on other excitations. This includes, but is not limited to, hybrid-phonon modes, Bogoliubov excitations in Bose-Einstein condensates, or the Jeans analysis of self-gravitating systems.

Of course, previous attempts to explain the variety of noncanonical distributions observed in plasma physics have been already considered in the literature from different perspectives. Those attempts can arguably be classified into two categories: on one hand, those focusing on very specific circumstances, for instance in the case of particles interacting with external radiation [76]; on the other hand, those attempting to explain these distributions in a more general context, mostly relying on generalized entropy functionals and nonextensive statistical mechanics. Both approaches face some limitations: While the former is restricted to systems under very special conditions, resulting in a narrow class of distributions with a limited range of allowable parameter values, the latter requires dealing with a free parameter, whose origin remains obscure, and a nonextensive entropy, possibly resulting in thermodynamic inconsistencies [75].

Other attempts at explaining the emergence of noncanonical distributions in space plasmas from statistical arguments include the approach put forward by Shizgal [36] that relies on the use of Fokker-Planck equations. Our approach is not opposed to but rather complements it. This raises questions such as how to link superstatistics to the Fokker-Planck equations and whether the class of lognormal distributions may arise from similar considerations (results in this direction, although in a different context, may be found in Ref. [24]).

Finally, note that we were not much concerned here in interpreting the formalism of superstatistics itself; our ambition is of a more practical nature. It should be noted however that by speaking about "fluctuations," we are tacitly 
adopting a frequentist interpretation of the formalism. That is, the temperature distribution is understood as representing temperature fluctuations that are actually taking place in the system. This may however not be taken too literally. One may alternatively adopt a Bayesian interpretation of the formalism [77], in which the temperature distributions do not correspond to fluctuations but rather express some kind of uncertainty. In this context, the so-called thermodynamic uncertainty relations (see for instance Ref. [14], pp. 195-199) may shed new light on the ubiquitous presence of noncanonical distributions in space plasmas.

\section{ACKNOWLEDGMENT}

I am particularly indebted to an anonymous referee for constructive critiques and insightful comments.
[1] G. Livadiotis and D. J. McComas, Astrophys. J. 741, 88 (2011).

[2] I. S. Elkamash and I. Kourakis, Phys. Rev. E 94, 053202 (2016).

[3] S. Sultana, R. Schlickeiser, I. S. Elkamash, and I. Kourakis, Phys. Rev. E 98, 033207 (2018).

[4] H. Fichtner, K. Scherer, M. Lazar, H. J. Fahr, and Z. Vörös, Phys. Rev. E 98, 053205 (2018).

[5] A. A. Mamun, Phys. Rev. E 55, 1852 (1997).

[6] A. A. Mamun and P. K. Shukla, Phys. Rev. E 80, 037401 (2009).

[7] F. Verheest, M. A. Hellberg, and I. Kourakis, Phys. Rev. E 87, 043107 (2013).

[8] A. A. Abid, S. Ali, J. Du, and A. A. Mamun, Phys. Plasmas 22, 084507 (2015).

[9] M. Tribeche, R. Amour, and P. K. Shukla, Phys. Rev. E 85, 037401 (2012).

[10] G. Williams, I. Kourakis, F. Verheest, and M. A. Hellberg, Phys. Rev. E 88, 023103 (2013).

[11] P. Jizba, J. Korbel, H. Lavička, M. Prokš, V. Svoboda, and C. Beck, Phys. A (Amsterdam) 493, 29 (2018).

[12] C. Beck and E. G. D. Cohen, Phys. A (Amsterdam) 322, 267 (2003).

[13] R. Kubo, M. Toda, and N. Hashitsume, Statistical Physics II: Nonequilibrium Statistical Mechanics (Springer, New York, 1995).

[14] B. H. Lavenda, Statistical Physics: A Probabilistic Approach (Wiley-Interscience, New York, 1991).

[15] G. Wilk and Z. Włodarczyk, Phys. Rev. Lett. 84, 2770 (2000).

[16] C. Beck, Phys. Rev. Lett. 87, 180601 (2001).

[17] F. Sattin and L. Salasnich, Phys. Rev. E 65, 035106(R) (2002).

[18] P. Jizba and H. Kleinert, Phys. Rev. D 82, 085016 (2010).

[19] A. Ayala, M. Hentschinski, L. A. Hernández, M. Loewe, and R. Zamora, Phys. Rev. D 98, 114002 (2018).

[20] P. Jizba and F. Scardigli, Eur. Phys. J. C 73, 2491 (2013).

[21] K. Ourabah, E. M. Barboza, Jr., E. M. C. Abreu, and J. A. Neto, Phys. Rev. D 100, 103516 (2019).

[22] M. Denys, T. Gubiec, R. Kutner, M. Jagielski, and H. E. Stanley, Phys. Rev. E 94, 042305 (2016).

[23] B. Schäfer, C. Beck, K. Aihara, D. Witthaut, and M. Timme, Nat. Energy 3, 119 (2018).

[24] A. V. Chechkin, F. Seno, R. Metzler, and I. M. Sokolov, Phys. Rev. X 7, 021002 (2017).

[25] K. Ourabah and M. Tribeche, Phys. Rev. E 95, 042111 (2017).

[26] K. Ourabah and M. Tribeche, Phys. Lett. A 381, 2659 (2017).

[27] K. Ourabah, L. Aït Gougam, and M. Tribeche, Phys. Rev. E 91, 12133 (2015).

[28] S. Davis, J. Jain, D. González, and G. Gutiérrez, J. Phys.: Conf. Ser. 1043, 012011 (2018).

[29] S. Davis, G. Avaria, B. Bora, J. Jain, J. Moreno, C. Pavez, and L. Soto, Phys. Rev. E 100, 023205 (2019).
[30] S. Davis, J. Phys. A 53, 045004 (2020).

[31] R. A. Cairns, A. A. Mamum, R. Bingham, R. Boström, R. O. Dendy, C. M. C. Nairn, and P. K. Shukla, Geophys. Res. Lett. 22, 2709 (1995).

[32] S. Olbert, in Physics of the Magnetosphere, Astrophysics and Space Science Library, Vol. 10, edited by R. D. L. Carovillano and J. F. McClay (D. Reidel, Dordrecht, 1968), p. 641.

[33] V. M. Vasyliūnas, J. Geophys. Res. 73, 2839 (1968).

[34] M. P. Leubner, Astrophys. Space Sci. 283, 573 (2002).

[35] G. Livadiotis and D. J. McComas, Space Sci. Rev. 175, 183 (2013).

[36] B. D. Shizgal, Phys. Rev. E 97, 052144 (2018).

[37] S. Nadarajah and S. Kotz, Phys. A (Amsterdam) 377, 465 (2007).

[38] C. Beck, E. G. D. Cohen, and H. L. Swinney, Phys. Rev. E 72, 056133 (2005).

[39] C. Tsallis, Introduction to Nonextensive Statistical Mechanics (Springer, New York, 2009).

[40] A. M. Reynolds, Phys. Rev. Lett. 91, 084503 (2003).

[41] S. Jung and H. L. Swinney, Phys. Rev. E 72, 026304 (2005).

[42] A. Y. Abul-Magd, B. Dietz, T. Friedrich, and A. Richter, Phys. Rev. E 77, 046202 (2008).

[43] K. Scherer, H. Fichtner, and M. Lazar, Europhys. Lett. 120, 50002 (2018).

[44] Š. Štverák, P. Trávníček, M. Maksimovic, E. Marsch, A. N. Fazakerley, and E. E. Scime, J. Geophys. Res. 113, A03103 (2008).

[45] M. I. Desai, G. M. Mason, M. A. Dayeh, R. W. Ebert, D. J McComas, G. Li, C. M. S. Cohen, R. A. Mewaldt, N. A. Schwadron, and C. W. Smith, Astrophys. J. 828, 106 (2016).

[46] G. Van Hoven, Phys. Rev. Lett. 17, 169 (1966).

[47] J. A. S. Lima, R. Silva, Jr., and J. Santos, Phys. Rev. E 61, 3260 (2000).

[48] R. Silva, J. S. Alcaniz, and J. A. S. Lima, Phys. A (Amsterdam) 356, 509 (2005).

[49] L. D. Landau, J. Phys. USSR 10, 25 (1946).

[50] P. M. Bellan, Fundamentals of Plasma Physics (Cambridge University Press, Cambridge, 2008).

[51] H. H. Kuehl, G. E. Stewart, and C. Yeh, Phys. Fluids 8, 723 (1965).

[52] E. Saberian and A. Esfandyari-Kalejahi, Phys. Rev. E 87, 053112 (2013); E. Saberian, ibid. 99, 017202 (2019).

[53] R. A. López, R. E. Navarro, S. I. Pons, and J. A. Araneda, Phys. Plasmas 24, 102119 (2017).

[54] G. Kaniadakis, Phys. Rev. E 66, 056125 (2002).

[55] J. A. S. Lima, R. Silva, and J. Santos, Astron. Astrophys. 396, 309 (2002).

[56] D. Jiulin, Phys. Lett. A 320, 347 (2004). 
[57] D. Jiulin, Phys. A (Amsterdam) 335, 107 (2004).

[58] M. Lazar, S. Poedts, and H. Fichtner, Astron. Astrophys. 582, A124 (2015).

[59] M. Lazar, H. Fichtner, and P. H. Yoon, Astron. Astrophys. 589, A39 (2016).

[60] M. Lazar and S. Poedts, Astron. Astrophys. 494, 311 (2009).

[61] G. Livadiotis, J. Geophys. Res. 120, 1607 (2015).

[62] S. Davis and G. Gutiérrez, Phys. A (Amsterdam) 505, 864 (2018).

[63] S. J. Bame et al., Astron. Astrophys., Suppl. Ser. 9, 237 (1992).

[64] M. Maksimovic, V. Pierrard, and P. Riley, Geophys. Res. Lett. 24, 1151 (1997).

[65] G. E. Uhlenbeck and G. W. Ford, Lectures in Statistical Mechanics (American Mathematical Society, Providence, 1963), p. 18.

[66] M. P. Almeida, Phys. A (Amsterdam) 300, 424 (2001).
[67] J. Lindhard, in The Lesson of Quantum Theory (North-Holland, Amsterdam, 1986).

[68] L. D. Landau and I. M. Lifschitz, Course of Theoretical Physics: Statistical Physics (Pergamon Press, New York, 1958).

[69] G. Wilk and Z. Włodarczyk, Phys. Rev. C 79, 054903 (2009).

[70] C. Kittel, Phys. Today 41(5), 93 (1988).

[71] B. B. Mandelbrot, Phys. Today 42(1), 71 (1989).

[72] J. Hickman and Y. Mishin, Phys. Rev. B 94, 184311 (2016).

[73] J. W. Gibbs, Elementary Principles in Statistical Mechanics (C. Scribner's Sons, New York, 1902).

[74] R. Balescu, Transport Processes in Plasmas (North-Holland, Amsterdam, 1988).

[75] M. Nauenberg, Phys. Rev. E 67, 036114 (2003).

[76] A. Hasegawa, K. Mima, and Minh Duong-van, Phys. Rev. Lett. 54, 2608 (1985).

[77] F. Sattin, Eur. Phys. J. B 49, 219 (2006). 